www.jmscr.igmpublication.org

Impact Factor 5.84

Index Copernicus Value: 71.58

ISSN (e)-2347-176x ISSN (p) 2455-0450

crossref DOI: _https://dx.doi.org/10.18535/jmscr/v5i9.35

Journal Of Medical Science And Clinical Research

\title{
Study of Serum Soluble Klotho in Egyptian Hemodialysis Patients
}

\section{Hesham K H Keryakos', Hisham Abdel Haleem Ali ${ }^{2}$, Aml Kamal Helmy $^{3}$, Mostafa Ahmed El-Sayed Abu-El-Ela ${ }^{4}$, Moustafa A. Kader A. Wahab ${ }^{5}$, TamerTaha Ismail ${ }^{6}$, Sherif R Abd Al-Razek ${ }^{7}$ \\ ${ }^{1}$ Division of Nephrology, Department of Internal Medicine, Faculty of Medicine, Minia, Egypt}

Email: keryakos.hesham@gmail.com

${ }^{2}$ Department of Internal Medicine, Faculty of Medicine, Minia, Egypt

Email: hishamali@yahoo.com

${ }^{3}$ Division of Nephrology, Department of Internal Medicine, Faculty of Medicine, Minia, Egypt

Email:abammo@yahoo.com

${ }^{4}$ Department of Clinical Pathology, Faculty of Medicine, Minia, Egypt

Email:mostafasayed@yahoo.com

${ }^{5}$ Department of Diagnostic Radiology, Faculty of Medicine, Minia, Egypt

Email: mostafakader@yahoo.com

${ }^{6}$ Department of Cardiology, Faculty of Medicine, Minia, Egypt

Email: tamertaha76@gmail.com

${ }^{7}$ Department of Internal Medicine, Minia, Egypt

Email: sherif@hotmail.com

\begin{abstract}
Preclinical studies demonstrated that klotho has cardio protective effect. One important biological function of Klotho is to maintain mineral homeostasis, which is both fibro blast growth factor-23 (FGF23)-dependent and FGF23-independent.

Aims: to study the relation between serum soluble klotho (sKlotho) and the following cardiovascular parameters; valvular calcification, vascular calcification, pulmonary hypertension (PH) and left ventricular hypertrophy (LVH). Also, we studied the relation between sklotho andbone mineral density (BMD) as well as serum ferritin.

Methods: 40 hemodialysis (HD) patients were recruited from Minia University Hospital dialysis center as well as 20 healthy controls participated in this study. sklotho, FGF-23, sclerostin, 25-OH-D, iPTH, and ferrit in were measured. High resolution peripheral quantitative computed tomography (HR-pQCT) was used to measure BMD. Multi-detector computed tomography (MDCT) was used to detect aortic calcification. Transthoracic echocardiography was used to detect $\mathrm{LVH}, \mathrm{PH}$, and valvular calcifications.

Results: sklotho level was significantly lower in HD patients than controls. In significant deffirences in sklotho levels between patients with and without PH and between patients with and without LVH. However, patients with higher sklotho had a trend toward protection from valvular and vascular calcification. There was a positive correlation between sklotho and 25-OH-D $(r=0.359, p=0.023)$, and serum corrected total calcium $(r=0.956, p<0.001)$. Mean while, there was a negative correlation between sklotho and BMD $(r=$
\end{abstract}


-0.425, $p=0.006), F G F-23(r=-0.375, p=0.017)$, sclerostin $(r=-0.445, p=0.004)$, phosphorus $(r=-$ $0.859, p<0.001)$. We found also that sklothois negatively correlated with serum ferritin in healthy controls and this correlation was disrupted in HD patients.

Conclusions: Higher sklotho was associated with decreased BMD, and a trend toward absence ofaortic and valvular calcifications in HD patients.

Keywords: sklotho, $P H, L V H$, aortic and valvular calcifications, BMD.

\section{Introduction}

Klotho was first identified in mice, where mutations of the gene led to a syndrome resembling aging ${ }^{[1]}$. The Klotho protein exists in both a membrane bound and a soluble form (sKlotho). sKlotho is derived from the extracellular part of membranous Klotho and through alternative splicing of the Klotho gene and has a unique role in renal calcium and phosphate excretion, independent of FGF$23^{[2,3,4,5]}$. Membrane-bound Klotho is a co-receptor for FGF-23 vital for FGF-23's phosphaturic effect. In chronic kidney disease (CKD) serum FGF-23 levels rise in response to phosphate retention, while Klotho levels decrease ${ }^{[6]}$. Elevated FGF-23 levels are associated with worse cardiovascular outcome but it is unknown whether this is due to the toxic effect of FGF-23 itself or due to Klotho deficiency causing a state of FGF-23 resistance ${ }^{[7,8]}$. sKlotho has been associated with protective effects on vascular calcification and oxidative stress in preclinical studies ${ }^{[9,10]}$. Since the kidneys are the principal source of sKlotho, some studies have suggested that CKD might be a state of progressive Klotho deficiency ${ }^{[11,12]}$. So we study the relation between sklotho and valvular calcification, vascular calcification, $\mathrm{PH}$ and $\mathrm{LVH}$ to investigateits cardioprotective effect. Also, we studied the relation between sklotho and BMD as well as serum ferritin to investigate if there cross-talk between iron homeostasis and chronic kidney disease - mineral bone disease (CKD-MBD).

\section{Subjects and Methods \\ Study Participants}

This study was an observational cross-sectional study of the relation between serum klotho and cardiovascular parameters of pulmonary hypertension, left ventricular hypertrophy, and aortic and valvular calcification; as well as its correlation with other biomarkers of CKD-BMD in hemodialysis patients; with the participants recruited from the hemodialysis unit of Minia University Hospital. The study enrolled Forty (40) patients with end-stage renal disease (ESRD) undergoing maintenance hemodialysis thrice weekly; as well as twenty (20) age- and sexmatched healthy controls. Informed consent was obtained from all participants. The inclusion criterion was end-stage renal disease patients on maintenance hemodialysis for at least 6 months. The exclusion criteria for ESRD patients were a history of pathologic fractures, established or suspected osteoporosis, past or present bisphosphonate therapy or known derangements of calcium homeostasis. The exclusion criteria for healthy controls were serum creatinine $(>1.2 \mathrm{mg} / \mathrm{dL})$ and iPTH serum levels ( $>65 \mathrm{pg} / \mathrm{mL})$. Plasma samples were collected at the beginning of the dialysis session, after a 2-day dialysis-free interval, and then stored at $-80^{\circ} \mathrm{C}$ until further analysis

\section{Primary Predictor and Outcome}

Serum klotho was measured by human Klotho ELISA kit, Catalogue number E2253h supplied from EIAab.www.eiab.com. The microtitre plate provided by the kit had been coated with an antibody specific to Klotho. Standards or samples were then added to the appropriate microtitre plate with a biotin-conjugated polyclonal ntibody preparation specific for Klotho, and Avidin conjugated to the Horseraddish peroxidase was added to each microplate well and incubated. Then a TMB substrate solution was added to each well. Only those wells that contain Klotho, biotin conjugated antibody and enzyme-conjugated Avidin exhibited a change in color. The enzyme - reaction was terminated by the addition of a sulfuric acid 
solution and the color change was measured spectrophotometrically at W.L. of $450 \mathrm{~nm}$. The concentration of Klotho in the sample was then determined by comparing the O.D. of the samples to the standard curve.

Cardiovascular parameters of pulmonary hypertension, left ventricular hypertrophy ( $\mathrm{LVH})$, and aortic and valvular calcifications as well as Bone mineral density were the outcomes selected for analysis. Bone mineral density was measured by high-resolution peripheral quantitative computed tomography (HR-pQCT). All patients were subjected to MDCT using 16-detector CT scanner (GE bright speed). Axial thin cut images were obtained on the dorsal and lumbosacral spines including the sternum, ribs and pelvic bones. Then, the axial images were reconstructed into coronal and sagittal images using separate workstation, after then we measured the CT density on the region of interest (ROI) having HU (Hounsfield unit) as a unit. We measured the HU of the vertebral body (medulla), sternum, ribs or sacrum (cortex, medulla) in our patients as well as our control group. Vascular calcification was evaluated using MDCT scan to detect the presence or absence of vascular calcification along the whole course of aorta on the thorax, abdomen if present; and transthoracic echocardiogram to detect the presence or absence of pulmonary hypertension (pulmonary arterial systolic pressure (PASP)> $35 \mathrm{mmHg}), L V H(\mathrm{LV}$ mass index was greater $>134 \mathrm{~g} / \mathrm{m} 2$ in men and $>110$ $\mathrm{g} / \mathrm{m} 2$ in Women), and valvular calcification.

\section{Other Measurements}

Demographic data, history of hypertension, diabetes, weight, height, BMI, and hemodialysis duration were obtained at enrollment. Complete blood count, serum albumin, calcium, phosphorus, urea, creatinine, and uric acid were measured using stored ethylenediaminetetraacetic acid (EDTA) plasma samples using standard techniques. CBC was obtained using Mindray BC-3200 auto hematology counter (Shenzhen Mindray BioMedical Electronics Co., Ltd. China). Serum albumin was measured using spectrophotometry. Serum calcium, phosphorus, urea, creatinine, and uric acid were measured using Dimension ES chemical auto-analyzer.

Plasma levels of serum were obtained using stored EDTA plasma samples. Measurements of plasma sclerostin concentrations were performed using a two-site second-generation enzyme-linked immunosorbent assay (ELISA) kit (EIAab Science Co. Ltd., Wuhan, China). The microtiter plate was coated with monoclonal anti-sclerostin antibody. $100 \mu \mathrm{l}$ of Standards or samples are added to the appropriate microtiter plate wells, and incubate for 2 hours at $37^{\circ} \mathrm{C}$. Remove the liquid of each well; add $100 \mu \mathrm{l}$ of a biotin-conjugated polyclonal antisclerostin antibody (Detection Reagent A) to each well and incubate for 1 hour at $37^{\circ} \mathrm{C}$. Aspirate each well and wash with wash buffer, repeating the process three times for a total of three washes, followed by the addition of $100 \mu$ of Avidin conjugated to Horseradish Peroxidase (HRP) (Detection Reagent B)to each microplate well and incubated for 1 hour at $37^{\circ} \mathrm{C}$. Color development was achieved using a $90 \mu \mathrm{TMB}$ substrate solution is added to each well and incubated for 30 minutes at $37^{\circ} \mathrm{C}$. Only those wells that contain, biotinconjugated antibody and enzyme-conjugated Avidin will exhibit a change in color. The enzyme-substrate reaction is terminated by the addition of $50 \mu \mathrm{l}$ sulphuric acid solution and the color change is measured spectrophotometrically at a wavelength of $450 \mathrm{~nm} \pm 2 \mathrm{~nm}$. Serial dilutions of recombinant human sclerostin were used to establish a standard curve. The intra assay and inter assay coefficient of variation was $\leq 5.3 \%$ and $\leq 8.4 \%$, respectively. Analytical measurement range for this assay was $0.83 \pm 0.22 \mathrm{ng} / \mathrm{ml}$ for males, $0.59 \pm 0.3 \mathrm{ng} / \mathrm{ml}$ for premenopausal females, and $0.66 \pm 0.22 \mathrm{ng} / \mathrm{ml}$ for postmenopausal females.

Plasma levels of 25-hydroxyvitamin D (25(OH)D) were obtained using stored EDTA plasma samples. Competitive protein binding immunoassay was used to measure 25(OH)D level (Immundiagnostik $A G$, Bensheim, Germany). Analytical measurement ranges for the $25(\mathrm{OH}) \mathrm{D}$ assay were $11-47 \mathrm{ng} / \mathrm{mL}$. The intra-assay coefficients of variations (CVs) 
were $10.7 \%$ at $4.5 \mathrm{nmol} / \mathrm{L}$. The inter-assay $\mathrm{CVs}$ were $13.2 \%$ and $11.8 \%$ at 39.8 and $86.7 \mathrm{nmol} / \mathrm{L}$, respectively. The Immunodiagnostic $25-\mathrm{OH}$ vitamin D total ELISA kit is a solid phase (enzyme-linked immunosorbent assay), based on the principle of competitive binding. In the first step, samples have to be pretreated in separate vials with precipitation reagent to extract the anylate, since most circulating $25-\mathrm{OH}$ vit $\mathrm{D}$ is bounded to vitamin $\mathrm{D}$ binding protein (VDBP) in vivo. In the first incubation step, sample, calibrator, control, the vitamin D binding protein and the VDBP-Antibody, an antibody specific for this protein is added to the solid phase. 25-OH Vit D present in the sample then competes with the tracer, coated on the well for the specific binding site of the binding protein and the VDBPAntibody is bound to the vitamin binding protein. Hence, with increasing concentrations of $25-\mathrm{OH}$ Vit $\mathrm{D}$ in the sample, the amount of binding protein, immobilized to the well via the tracer, is reduced. After a washing step to remove unbound components, the quantitation of VDBP is achieved by incubation with a host specific peroxidase labeled antibody using TMB (tetramethylbenzidine) as enzyme substrate. An acidic stopping solution is then added to stop the reaction. The color converts to yellow. The intensity of the yellow color is indirectly proportional to the concentration of 25$\mathrm{OH}$ Vitamin D in the sample. A dose response curve of the absorbance unit vs. concentration is generated using the results obtained from the calibrators. Concentrations of 25-OH Vitamin D, present in the patient samples, are determined directly from this curve.

Plasma levels of intact parathyroid hormone (iPTH) were obtained using stored EDTA plasma samples. iPTH was measured using a solid phase Enzyme Amplified Sensitivity Immunoassay performed on micro titer plates (DIA source Immuno Assays S.A.Belgium) with a reference interval of $12-72 \mathrm{pg} / \mathrm{mL}$. The intra-assay coefficients of variations (CVs) were $1.1 \%$ and $2.0 \%$ at $41.0 \pm 0.5$ and $594 \pm 12$ $\mathrm{pg} / \mathrm{mL}$ respectively. Inter-assay $\mathrm{CVs}$ were $7.1 \%$ and $45.7 \pm 3.3$ and $381 \pm 11.1 \mathrm{pg} / \mathrm{mL}$ respectively. iPTH assay was performed according to the following steps; $100 \mu \mathrm{l}$ of incubation buffer are added to all wells, and add $200 \mu \mathrm{l}$ of each calibrator, control and sample into appropriate wells. Calibrators and samples react with the capture polyclonal antibodies (PAb, goat anti 1-34 PTH fragment) coated on microtiter well. Incubate for 2 hours at room temperature on a horizontal shaker set at $700 \mathrm{rpm} \pm$ $100 \mathrm{rpm}$. Aspirate the liquid from each well; then wash the plate 4 times by wash solution to remove the excess antigen. Add $100 \mu \mathrm{l}$ of monoclonal antibodies (MAb, mouse anti 44-68 PTH fragment) labelled with horseradish peroxidase (HRP) into all the wells, and incubate for 1 hour at room temperature on a horizontal shaker set at $700 \mathrm{rpm} \pm$ $100 \mathrm{rpm}$ allowing the formation of a sandwich: coated PAbs-human PTH-MAb-HRP. Aspirate the liquid from each well; then wash the plate 4 times by wash solution to remove unbound enzyme labelled antibody. Bound enzyme-labelled antibody is measured through a chromogenic reaction. $100 \mu \mathrm{l}$ of the Chromogenic Solution (TMB) is added to each well within 15 minutes following the washing step, and incubate for 30 minutes at room temperature on a horizontal shaker set at $700 \mathrm{rpm} \pm$ $100 \mathrm{rpm}$. The reaction is terminated by the addition of $200 \mu \mathrm{l}$ of Stop Solution and the color change is read at the appropriate wavelength of $450 \mathrm{~nm}$ and $490 \mathrm{~nm}$ within 1 hour. The amount of substrate turnover is determined calorimetrically by measuring the absorbance, which is proportional to the PTH concentration.

\section{Statistical Analysis}

All statistical analyses were performed with Statistical Package for the Social Science (SPSS for windows version 20.0) (SPSS Inc., Tokyo, Japan). The continuous variables were expressed as mean \pm SD which compared using chi-square test. Statistical significance was defined as a probability level of $p<0.05$. 


\section{Results}

Serum Klotho levels in dialysis patients and healthy controls

Demographic data of dialysis patients are given in Table 1. This table shows that there is no significant difference $(p<0.05)$ between both groups as regard age, weight, height BMI and sex.

Table 1. Demographic data in hemodialysis patients and healthy controls:

\begin{tabular}{|l|c|c|c|}
\hline & $\begin{array}{c}\text { Cases } \\
(\mathrm{n}=40)\end{array}$ & $\begin{array}{c}\text { Control } \\
(\mathrm{n}=20)\end{array}$ & $p$ value \\
\hline Age (years) & $41.5 \pm 14.82$ & $40.4 \pm 13.94$ & 0.852 \\
\hline Sex: & $23(57.5 \%)$ & $12(60 \%)$ & 0.853 \\
Male $(\%)$ & $17(42.5 \%)$ & $8(40 \%)$ & 0.756 \\
Female $(\%)$ & $62.15 \pm 12.34$ & $61.05 \pm 15.36$ & 0.766 \\
\hline Weight $(\mathrm{Kg})$ & $1.61 \pm 0.78$ & $1.59 \pm 0.08$ & 0.452 \\
\hline Height $(\mathrm{m})$ & $23.88 \pm 3.91$ & $23.74 \pm 4.46$ & 0.957 \\
\hline BMI $\left(\mathrm{kg} / \mathrm{m}^{2}\right)$ & & & \\
\hline
\end{tabular}

Serum levels of sklotho, sclerostin, iPTH, corrected calcium, phosphorus, 25-OH- D, hemoglobin, and ferritin in hemodialysis patients and healthy controls are shown in Table 2. This table shows that there was no significant difference between the dialysis patients and control group as regards $25(\mathrm{OH})-\mathrm{D}(p>0.05)$, while there was a significant decrease in the hemoglobin level, serum corrected total calcium and serum sklotho in hemodialysis patients $(p<0.05)$. Also there was a significant increase in the level of serum levels of sclerostin, iPTH, ferritin and phosphorus in hemodialysis patients as compared to the control group $(p<0.05)$.

Table 2. Intact PTH, serologic bone markers, sclerostin, and sklotho in hemodialysis patients (HD) and healthy controls

\begin{tabular}{|l|c|c|c|}
\hline & $\begin{array}{c}\text { HD }(\text { mean } \pm \text { SD) } \\
(\mathrm{n}=40)\end{array}$ & $\begin{array}{c}\text { Control (mean } \pm \text { SD) } \\
(\mathrm{n}=20)\end{array}$ & $p$ value \\
\hline Sclerostin $(\mathrm{ng} / \mathrm{ml})$ & $0.93 \pm 1.72$ & $0.74 \pm 0.18$ & $<0.001$ \\
\hline iPTH $(12-72 \mathrm{pg} / \mathrm{ml})$ & $451.03 \pm 497.7$ & $38.15 \pm 17.56$ & $<0.001$ \\
\hline $25-($ OH-D $(11-47 \mathrm{ng} / \mathrm{ml})$ & $42.59 \pm 33.71$ & $30.85 \pm 10.61$ & 0.643 \\
\hline Corrected Calcium $(\mathrm{mg} / \mathrm{dl})$ & $8.14 \pm 0.23$ & $10.16 \pm 0.55$ & $<0.001$ \\
\hline Phosphorus $(\mathrm{mg} / \mathrm{dl})$ & $6.31 \pm 1.08$ & $4.11 \pm 0.5$ & $<0.001$ \\
\hline Hemoglobin $(\mathrm{g} / \mathrm{dl})$ & $9.99 \pm 1.77$ & $12.68 \pm 0.94$ & $<0.000$ \\
\hline Serum ferritin $(\mathrm{ng} / \mathrm{ml})$ & $\begin{array}{c}457.95 \pm 254.95 \\
(105-982)\end{array}$ & $\begin{array}{c}84.5 \pm 64.56 \\
(17-251)\end{array}$ & $<0.000$ \\
\hline Serum sklotho $(\mathrm{ng} / \mathrm{ml})$ & $\begin{array}{c}5.17 \pm 2.07 \\
(1-9)\end{array}$ & $\begin{array}{c}133.95 \pm 85.63 \\
(38-384)\end{array}$ & $<0.000$ \\
\hline
\end{tabular}

Relation between serum klotho and According to the presence or absence of LVH cardiovascular parameters

According to the presence or absence of pulmonary hypertension

- Group I: presence of pulmonary hypertension; included 25 patients.

- Group II: absence of pulmonary hypertension; included 15 patients.

Mean serum level of sklotho showed no statistical significance $(p$ value $=0.815)$ (Table 3$)$.

Table 3 Mean serum level of klotho in hemodialysis patients with and without pulmonary hypertension.

- Group I: presence of $\mathrm{LVH}$; included 31 patients.

- Group II: absence of LVH; included 9 patients.

Mean serum level of sklotho showed no statistical significance $(p$ value $=0.133)($ Table 4$)$.

Table 4 Mean serum level of sklotho in hemodialysis patients with and without LVH.

\begin{tabular}{|l|c|c|c|}
\hline \multirow{2}{*}{ Serum sklotho } & \multicolumn{2}{|c|}{ Left ventricular hypertrophy } & \multirow{2}{*}{$p$ value } \\
\cline { 2 - 3 } & Yes $(\mathrm{n}=31)$ & No $(\mathrm{n}=9)$ & \\
& $4.89 \pm 1.76$ & $6.08 \pm 2.84$ & 0.133 \\
\hline
\end{tabular}

\begin{tabular}{|l|c|c|c|}
\hline \multirow{2}{*}{} & \multicolumn{2}{|c|}{ Pulmonary hypertension } & \multirow{2}{*}{$p$ value } \\
\cline { 2 - 3 } & Yes & No & \\
\hline Serum & $5.11 \pm 1.76$ & $5.26 \pm 2.57$ & \multirow{2}{*}{0.815} \\
\hline sKlotho & $(1.18-8.8)$ & $(3.8-7.5)$ & \\
\hline
\end{tabular}


According to the presence or absence of valve calcification:

- Group I: presence of valve calcification; included 8 patients.

- Group II: absence of valve calcification; included 32 patients.

Mean serum level of sklotho showed no statistical significance but higher levels showed a trend toward protection from valve calcification ( $p$ value $=0.068)$ (Table 5).

Table 5. Mean serum level of sklotho in hemodialysis patients with and without valve calcification.

\begin{tabular}{|l|c|c|c|}
\hline & \multicolumn{2}{|c|}{ Valve Calcification } & \multirow{2}{*}{$p$ value } \\
\cline { 2 - 3 } & Yes $(\mathrm{n}=8)$ & No $(\mathrm{n}=32)$ & \\
\hline Serum sklotho & $\begin{array}{l}3.2 \pm 1.35 \\
(1.18-5)\end{array}$ & $\begin{array}{c}4.4 \pm 1.73 \\
(1.18-8.8)\end{array}$ & \multirow{2}{*}{0.068} \\
\hline
\end{tabular}

According to the presence or absence of vascular calcification:

- Group I: presence of vascular calcification; included 19 patients.

- Group II: absence of vascular calcification; included 21 patients.

Mean serum level of klotho showed no statistical significance but higher levels showed a trend toward protection from vascular calcification ( $p$ value $=0.078)($ Table 6) .

Table 6. Mean serum level of sklotho in hemodialysis patients with and without vascular calcification.

\begin{tabular}{|l|c|c|c|}
\hline \multirow{2}{*}{ Serum sklotho } & \multicolumn{2}{|c|}{ Vascular Calcification } & \multirow{2}{*}{ v value } \\
\cline { 2 - 3 } & $\begin{array}{c}3.73 \pm 1.35 \\
(1.18-7.5)\end{array}$ & $\begin{array}{c}4.7 \pm 1.97 \\
(1.18-8.8)\end{array}$ & \multirow{2}{*}{0.078} \\
\hline
\end{tabular}

Correlation between serum klotho and serum ferritin with pulmonary artery systolic pressure (PASP), BMD, serum FGF-23, serum sclerostin, serum 25-OH-D, serum iPTH, corrected total calcium and phosphorus:

There was a positive correlation between serum sklotho and serum 25-OH-D ( $r=0.359, p=0.023)$, and corrected total calcium $(r=0.956, p=<0.001)$. Meanwhile, there was a negative correlation between serum sklotho and BMD $(r=-0.425, p=$
0.006), FGF-23 ( $r=-0.375, p=0.017)$, serum sclerostin $(r=-0.445, p=0.004)$, serum phosphorus ( $r=-0.859, p=<0.001)$. There was no correlation between serum ferritin and BMD, serum FGF-23, serum sclerostin, serum 25-OH-D, serum iPTH, corrected total calcium, and serum phosphorus (Table 7).

Table 7. Correlation results between serum sklotho and serum ferritin with PASP, BMD, serum FGF23, serum sclerostin, serum 25-OH-D, serum iPTH, corrected total calcium and phosphorus

\begin{tabular}{|l|c|c|c|c|}
\hline \multirow{2}{*}{} & \multicolumn{2}{|c|}{ Serum s } & \multicolumn{2}{c|}{ Serum Ferritin } \\
\cline { 2 - 5 } & $\mathrm{r}$ & $p$ value & $\mathrm{R}$ & $p$ value \\
\hline PASP & -0.034 & 0.834 & -0.155 & 0.339 \\
\hline BMD & -0.425 & $0.006^{*}$ & 0.088 & 0.588 \\
\hline Sclerostin & -0.375 & $0.017^{*}$ & 0.066 & 0.687 \\
\hline $25-$ OH-D & -0.445 & $0.004^{*}$ & -0.105 & 0.521 \\
\hline$i P T H$ & 0.359 & $0.023^{*}$ & -0.065 & 0.689 \\
\hline $\begin{array}{l}\text { Corrected Total } \\
\text { calcium }\end{array}$ & -0.259 & 0.107 & 0.057 & 0.725 \\
\hline Phosphorus & 0.956 & $<0.001^{*}$ & -0.044 & 0.787 \\
\hline
\end{tabular}

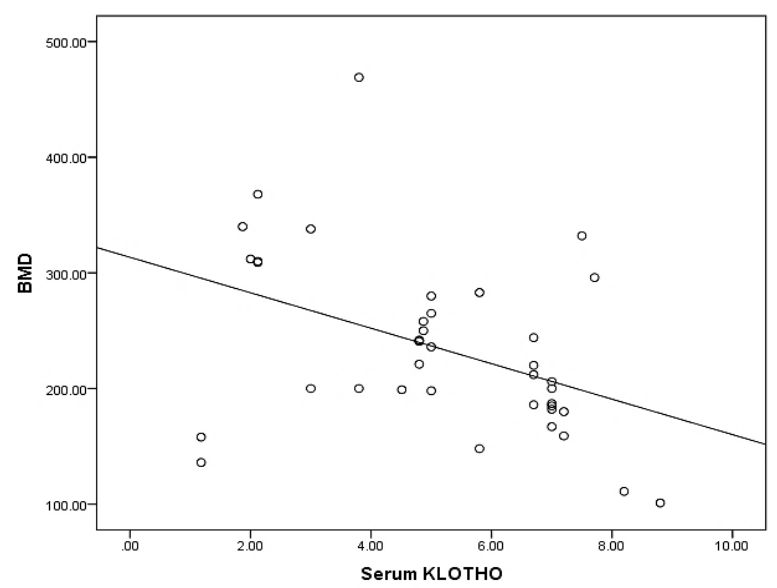

Figure2. Correlation between serum sklotho and $\mathrm{BMD}$ in hemodialysis patients

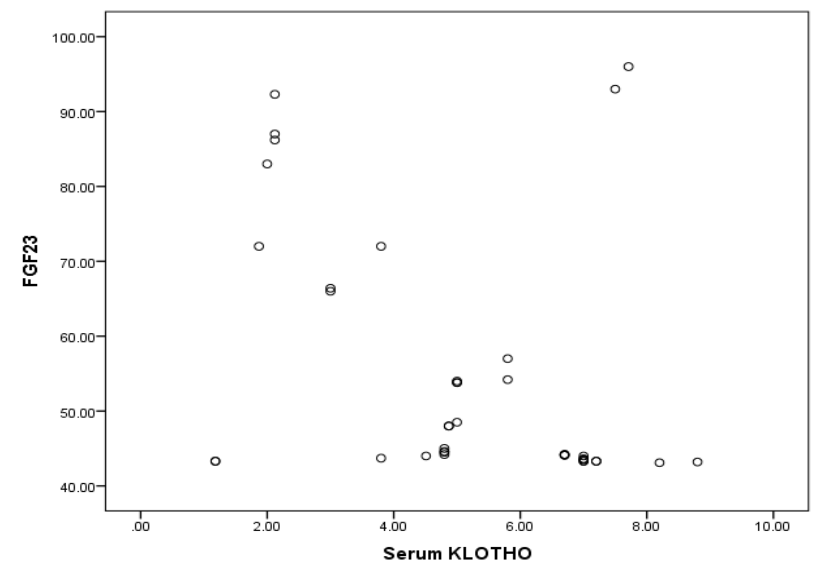

Figure 3. Correlation between serum sklotho and FGF-23 in hemodialysis patients 


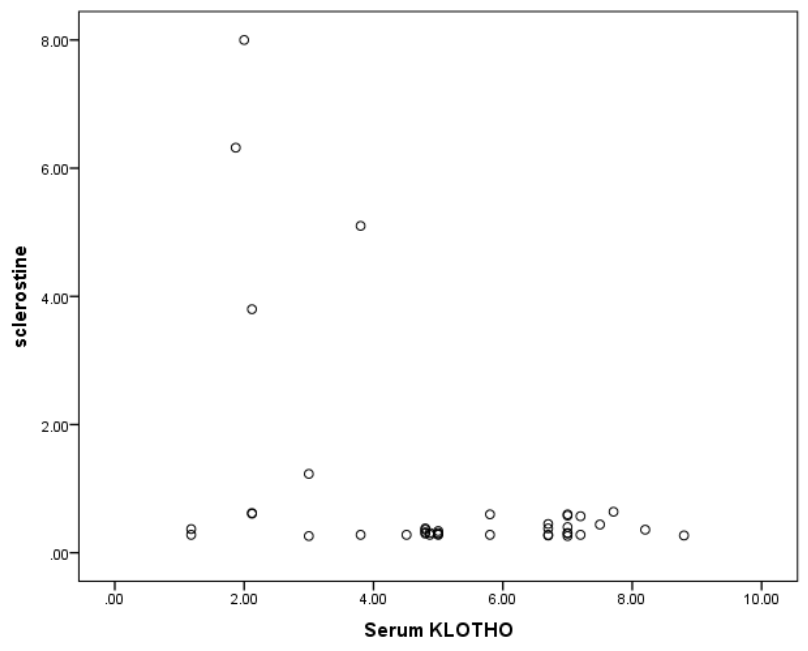

Figure 4.Correlation between serum sklotho and serum sclerostin

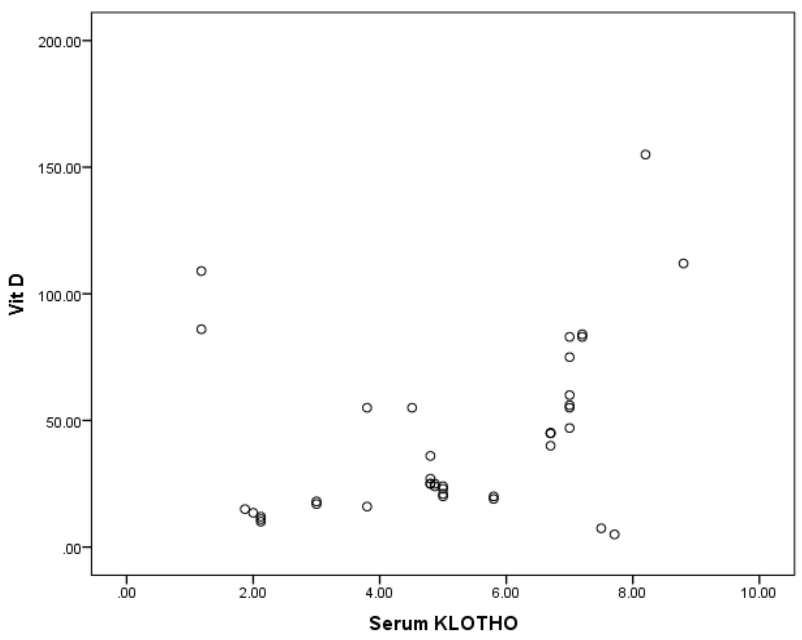

Figure 5.Correlation between serum sklotho and 25-OH-D in hemodialysis patients

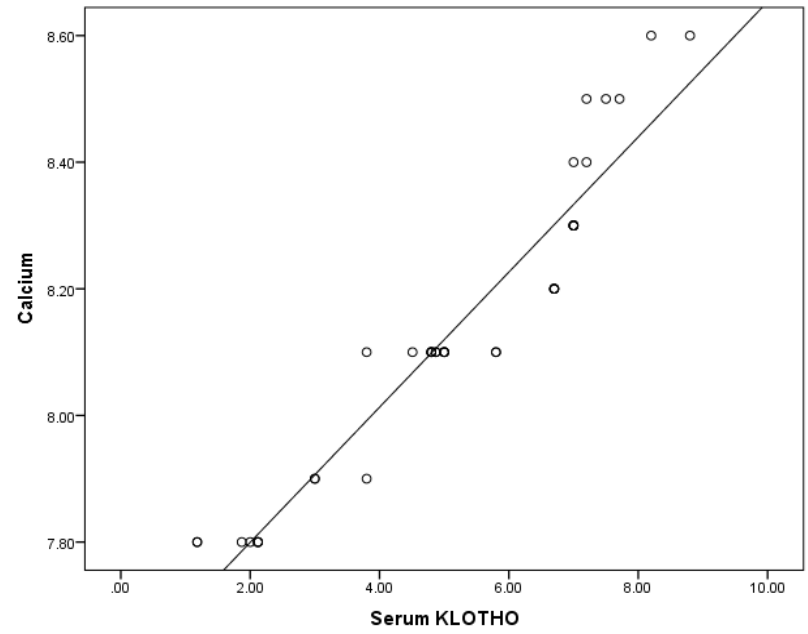

Figure 6. Correlation between serum sklotho and corrected calcium in hemodialysis patients

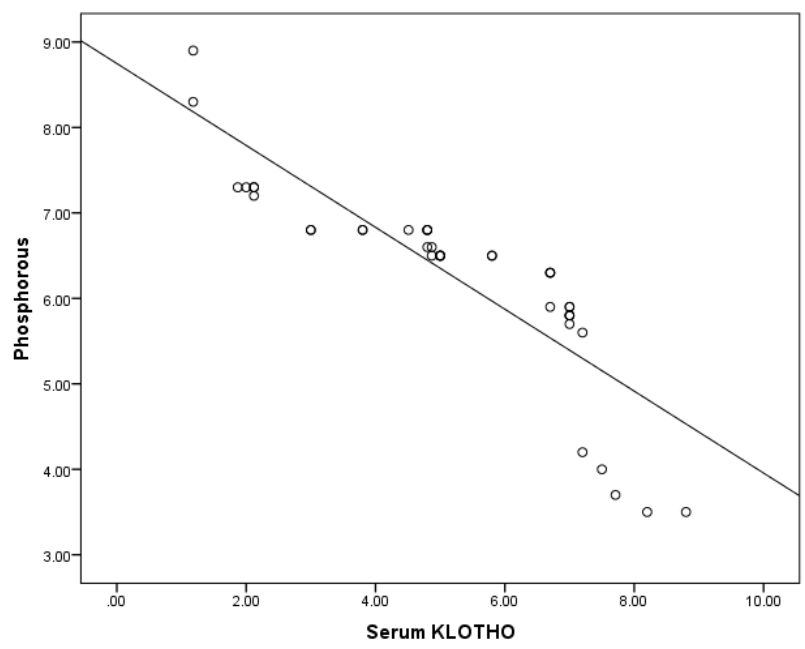

Figure 7. Correlation between serum sklotho and phosphorus in hemodialysis patients

\section{Correlation between serum klotho and serum ferritin}

There was a negative correlation between serum ferritin and serum sklotho in hemodialysis patients but not statistically significant, however we found negative correlation in healthy controls (Table 8 and Figure 8).

Table 8: Correlation between serum sklotho and serum ferritin

\begin{tabular}{|c|c|c|c|c|}
\hline & \multicolumn{2}{|c|}{ Hemodialysis Patients } & \multicolumn{2}{|c|}{ Healthy Controls } \\
\hline & \multicolumn{4}{|c|}{ Serum sKlotho } \\
\hline & $\mathrm{r}$ & $p$ value & $\mathrm{R}$ & $p$ value \\
\hline $\begin{array}{l}\text { Serum } \\
\text { ferritin }\end{array}$ & -0.273 & 0.145 & -0.543 & 0.002 \\
\hline
\end{tabular}

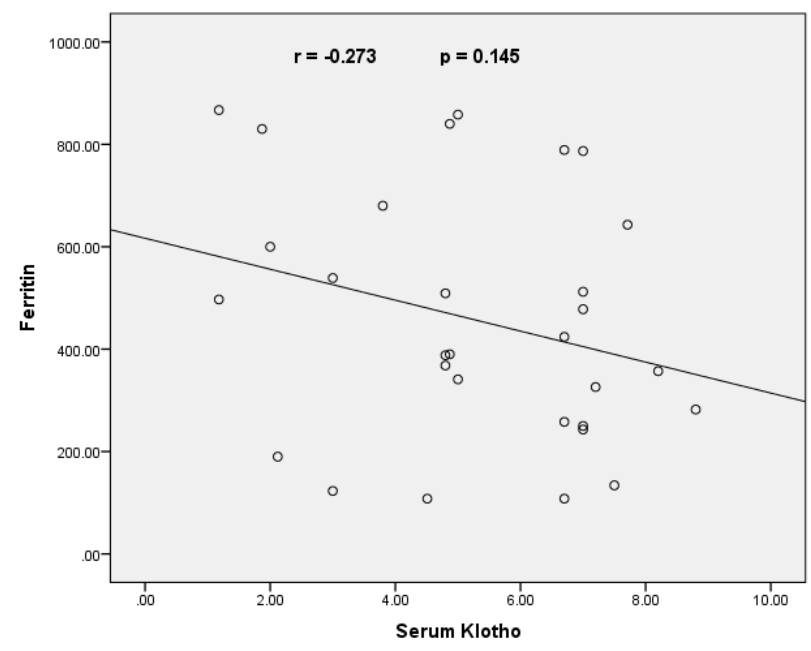

Figure 8: Correlation between serum sklotho and serum ferritin in healthy controls 


\section{Discussion}

Our cross-sectional observational study has demonstrated that circulating sklotho levels are lower in hemodialysis patients than healthy controls. Secondly, patients with higher serum sklotho levels showed a trend toward protection from valvular and vascular calcification. Thirdly, serum sklotho was negatively correlated with BMD, FGF-23, sclerostin, and phosphorus; with positive correlation with 25-OH-D. Finally, we found that serum sklotho is negatively correlated with serum ferritin and this correlation was disrupted in hemodialysis patients.

In the present study, serum sklotho levels were lower in hemodialysis patients than in healthy persons. Klotho has been documented to be decreased in various tissues in patients with CKD, and reduced Klotho expression within the parathyroid tissue has been demonstrated in advanced CKD patients. The vascular smooth muscle also appears to be Klotho-deficient in patients with $\mathrm{CKD}^{[13]}$. The kidney is a major source of circulating sKlotho as demonstrated by $30 \%$ to $50 \%$ decreases after nephrectomy in living donors in humans without deteriorated renal function ${ }^{[14]}$, and also recently in animal studies ${ }^{[15]}$. Moreover, recent demonstrations of Klotho expression in vascular tissue highlight the vascular territory as another source of sKlotho ${ }^{[10]}$.

In one prospective study, they found that hemodialysis patients with lower serum klotho levels had significantly higher cardiovascular and all-cause mortality rates ${ }^{[16]}$. In the present study, we investigate the correlation between serum sKlotho level and four different cardiovascular parameters; pulmonary hypertension (PH), left ventricular hypertrophy (LVH), vascular calcification, and valvular calcification.

$\mathrm{PH}$ has been recognized as a common complication of CKD and end-stage renal disease (ESRD).There is significant epidemiological overlap with kidney disease and the underlying causes of World Health Organization group 1-4 pulmonary hypertension (pulmonary arteriopathy, left heart disease, chronic pulmonary disease, and chronic thromboembolic disease, respectively). In addition, an entity of 'unexplained pulmonary hypertension', group 5, in patients with chronic kidney disease and end-stage renal disease has emerged, with prevalence estimates of $30-50 \%$. The pathogenesis of pulmonary hypertension in this population is due to alterations in endothelial function, increased cardiac output, and myocardial dysfunction leading to elevated left heart filling pressure, with recent data suggesting that left heart dysfunction may account for the vast majority of pulmonary hypertension in patients with kidney disease. Pulmonary hypertension is an independent predictor of increased mortality in patients on dialysis and those undergoing kidney transplantation ${ }^{[17]}$. As regard $\mathrm{LVH}$, it has been shown that serum sKlotho does not seem to be expressed in the left ventricle, and FGF-23 induced LVH in patients with ESRD was found to be klotho-independent ${ }^{[18]}$. We studied the correlation between serum klotho levels and both pulmonary hypertension and $\mathrm{LVH}$, and we found that patients with lower serum klotho levels showing a trend toward presence of pulmonary hypertension and LVH. Our findings are in agreement with a recently published study that showed that patients with a low sKlotho were more often associated with increased Carotid intimamedia thickness and LV dysfunction ${ }^{[19]}$.

An association between klotho deficiency and vascular calcification has been reported in aging mice and in a mouse model of $\mathrm{CKD}^{[1,13]}$. Klotho deficiency predisposed vascular smooth muscle cells (VSMCs) to transform into osteoblast-like cells that are ready to initiate mineralization in response to phosphate uptake. These findings are confirmed in human VCMC ${ }^{[20]}$. So Klotho is protective against vascular calcification by preventing differentiation of the VSMC to an osteoblast-like phenotype. The present study showed that patients with lower serum klotho levels had a trend toward the development of valve and vascular calcification but not reaching statistical significance.

In our study, there was a negative correlation between serum klotho and BMD, serum FGF-23, 
serum sclerostin, and serum phosphorus. The negative relationship between s-Klotho and serum $\mathrm{P}$ is interesting due to the reported direct effect of circulating s-Klotho on the renal expression of NaPi2a. Since s-Klotho and FGF23 correlate negatively in CKD but exert a similar positive effect on renal expression of $\mathrm{NaPi} 2 \mathrm{a}$, it is possible that the action of s-Klotho is shadowed by FGF23. The negative correlation between sKlotho and FGF-23 favorsthe hypothesis that s-Klotho, whose circulating levels are strongly related to renal function and are most probably secondary to reduced expression of transmembrane Klotho (TMKlotho), can be regarded as a sensitive biomarker of TM-Klotho expression, useful to appreciate early development of tubular resistance to FGF23. Consequently, bone synthesis of FGF23 is increased. A recent paper with histologic data from patients with glomerulonephritis showed parallel reduction of renal Klotho and of s-Klotho together with increments in FGF23, which is in agreement with our data ${ }^{[20]}$. We found that lower s-Klotho is associated with better bone mineral density and higher serum sclerostin. The correlation between serum sclerostin and bone mineral density is documented but the relationship between serum sKlotho and serum sclerostin has not yet studied ${ }^{[21]}$. Meanwhile, there is a positive correlation between serum sklotho and 25-OH-D, and corrected total calcium. This is consistent with many studies in CKD patients ${ }^{[19,22]}$

Limitations of our study are several. First, the number of evaluated patients is rather low. In fact, an increased number of cases would have reinforced the reliability of our results that, however, are in line with published data in the literature. Second, we did not prove that circulating s-Klotho reflect transmembrane Klotho.

Our study should spark interest into research aimed at further elucidating additional mechanisms by which soluble sklotho affect bone turnover and cardiovascular mortality risk in hemodialysis patients; and whether these findings are also working in CKD stages 3-4. Also, more research is needed to investigate the link between iron homeostasis and CKD-MBD.

\section{References}

1. Kuro OM, Matsumura $\mathrm{Y}$, Aizawa $\mathrm{H}$, Kawaguchi H, Suga T, Utsugi T, Ohyama Y, Kurabayashi M, Kaname T, Kume E, Iwasaki H, Iida A, Shiraki-Iida T, Nishikawa S, Nagai R, Nabeshima YI: Mutation of the mouse klotho gene leads to a syndrome resembling ageing. Nature.1997, 390: 45-51.

2. Imura A, Iwano A, Tohyama O, Tsuji Y, Nozaki K, Hashimoto N, Fujimori T, Nabeshima Y: Secreted Klotho protein in sera and CSF: implication for posttranslational cleavage in release of Klotho protein from cell membrane. FEBS Lett. 2004, 565: 143-147.

3. Matsumura Y, Aizawa H, Shiraki-Iida T, Nagai R, Kuro-o M, Nabeshima Y: Identification of the human klotho gene and its two transcripts encoding membrane and secreted klotho protein. Biochem Biophys Res Commun. 1998, 242: 626-630.

4. Chang Q, Hoefs S, van der Kemp AW, Topala CN, Bindels RJ, Hoenderop JG: The beta-glucuronidase klotho hydrolyzes and activates the TRPV5 channel. Science. 2005, 310: 490-493.

5. Hu MC, Shi M, Zhang J, Pastor J, Nakatani T, Lanske B, Razzaque MS, Rosenblatt KP, Baum MG, Kuro-o M, Moe OW: Klotho: a novel phosphaturic substance acting as an autocrine enzyme in the renal proximal tubule. FASEB J. 2010, 24: 3438-3450.

6. Pavik I, Jaeger P, Ebner L, Wagner CA, Petzold K, Spichtig D, Poster D, Wuthrich RP, Russmann S, Serra AL: Secreted Klotho and FGF23 in chronic kidney disease Stage 1 to 5: a sequence suggested from a crosssectionalstudy. Nephrol Dial Transplant. 2013, 28: 352-359.

7. $\mathrm{Hu}$ MC, Shi M, Zhang J, Quinones H, Griffith C, Kuro- OM, Moe OW: Klotho deficiency causes vascular calcification in 
chronic kidney disease. J Am Soc Nephrol. 2011, 22: 124-136.

8. Seiler S, Wen M, Roth HJ, Fehrenz M, Flugge F, Herath E, Weihrauch A, Fliser D, Heine GH: Plasma Klotho is not related to kidney function and does not predict adverse outcome in patients with chronic kidney disease. Kidney Int. 2013, 83: 121-128.

9. Yamamoto M, Clark JD, Pastor JV, Gurnani P, Nandi A, Kurosu H, Miyoshi M, Ogawa Y, Castrillon DH, Rosenblatt KP, Kuro-o M: Regulation of oxidative stress by the antiaging hormone klotho. J Biol Chem. 2005, 280: 38029-38034.

10. Lim K, Lu TS, Molostvov G, Lee C, Lam FT, Zehnder D, Hsiao LL: Vascular Klotho deficiency potentiates the development of human artery calcification and mediates resistance to fibroblast growth factor 23 . Circulation. 2012, 125: 2243-2255.

11. Hu MC, Shiizaki K, Kuro-o M, Moe OW: Fibroblast growth factor 23 and Klotho: physiology and pathophysiology of an endocrine network of mineral metabolism. Annu Rev Physiol. 2013, 75: 503-533.

12. Lindberg K, Amin R, Moe OW, Hu MC, Erben RG, Ostman WA, Lanske B, Olauson $\mathrm{H}$, Larsson TE: The kidney is the principal organ mediating klotho effects. J Am Soc Nephrol. 2014, 25: 2169-2175.

13. Hu MC, Shi M, Zhang J, Quinones H, Griffith C, et al. Klotho deficiency causes vascular calcification in chronic kidney disease. J Am Soc Nephrol.2011,22: 124136.

14. Akimoto T., Kimura T., Watanabe Y. et al., The impact of nephrectomy and renal transplantation on serum levels of soluble Klotho protein. Transplantation Proceedings. 2013; 45: 134-136.

15. Hu MC, Shi M, Zhang J et al., Renal production, uptake, and handling of circulating $\alpha$ Klotho. J Am Soc Nephrol. 2016; 27(1):79-90.
16. Naoko OT, Takahiro M, Tetsu A, Sumiko H, Yuko W, Kazuhiro S, Takuya M, Eiji K, Yasushi A, Makoto K, and Daisuke N. Association between Serum Soluble Klotho Levels and Mortality in Chronic Hemodialysis Patients. International Journal of Endocrinology 2015, Article ID 406269, 10 pages

17. Sise ME, Courtwright AM and Channick RN. Pulmonary hypertension in patients with chronic and end-stage kidney disease Kidney International (2013) 84, 682-692.

18. Faul C, Amaral AP, Oskouei B et al. FGF23 induces left ventricular hypertrophy. J Clin Invest 2011; 121: 4393-4408

19. Abdallah E, Mosbah O, Khalifa G, Metwaly A, El-Bendary O. Assessment of the relationship between serum soluble Klotho and carotid intimaemedia thickness and left ventricular dysfunction in hemodialysis patients Kidney Res Clin Pract 2016; 35:4249

20. Sakan H, Nakatani K, Asai O, Imura A, Tanaka T, Yoshimoto S, Iwamoto N, Kurumatani N, Iwano M, Nabeshima $Y$, Konishi N, Saito Y. Reduced renal $\alpha$-Klotho expression in CKD patients and its effect on renal phosphate handling and vitamin $\mathrm{D}$ metabolism. PLoS One. 2014. 23;9(1):e 86301.

21. Paccou J, Mentaverri R, Renard C, Liabeuf S, Fardellone P, Massy ZA, Brazier M, Kamel S. The relationships between serum sclerostin, bone mineral density, and vascular calcification in rheumatoid arthritis. J Clin Endocrinol Metab. 2014; 99(12):4740-4748.

22. Sawires HK, Essam RM, Morgan MF, Mahmoud RA: Serum klotho: relation to fibroblast growth factor-23 and other regulators of phosphate metabolism in children with chronic kidney disease. Nephron.2015, 129:293-299. 